\section{ESTIMATIVAS E TENDÊNCIAS DE ABORTO PROVOCADO NO PIAUÍ: UM ESTUDO ECOLÓGICO NO PERÍODO DE 2000-2010}

\author{
Estimates and trends of induced abortion in Piauí: an \\ ecological study of the period 2000-2010
Estimación y tendencias de aborto provocado en Piauí: un estudio ecológico en el período de 2000-2010

\section{RESUMO}

Objetivo: Estimar o aborto provocado e avaliar sua tendência no estado do Piaú e em seus territórios de desenvolvimento (TD), no período de 2000 a 2010. Métodos: Realizou-se estudo ecológico, de série temporal, utilizando dados secundários de internações hospitalares por complicações de aborto, no período de fevereiro a junho de 2014. A estimativa de abortos induzidos foi calculada por metodologia do Instituto Guttmacher. Empregou-se o método Joinpoint para identificar mudanças significativas nas tendências. Resultados: Analisaramse 55.678 internações hospitalares por aborto e verificou-se decréscimo de $35 \%$ entre 2000 e 2010. O número de abortos induzidos decresceu no Piauí entre os extremos da série temporal, passando de $10.362 \mathrm{em} 2000$ para $6.738 \mathrm{em} \mathrm{2010.} \mathrm{Houve} \mathrm{tendência} \mathrm{de} \mathrm{aumento} \mathrm{significativo}$ nas razões de aborto nos TD 1 e 7, como também tendência de redução significativa no TD 4. Os demais TD apresentaram tendência estável da razão de aborto. Conclusão: A taxa de internação pós-aborto e as estimativas de aborto induzido foram elevadas no estado do Piauí, com tendência de diminuição da razão de aborto induzido no período estudado.

Descritores: Aborto Induzido; Hospitalização; Estudos de Séries Temporais.

\section{ABSTRACT}

Objective: To estimate the induced abortion and assess its trend in the state of Piaui state and its development territories (DTs) in the period 2000-2010. Methods: We conducted an ecological time-series study using secondary data from hospital admissions for abortion complications in the period from February to June 2014. Induced abortions was estimated using the Guttmacher Institute methodology. The Joinpoint method was used to identify significant changes in trends. Results: 55,678 admissions for abortion were analyzed; there was a 35\% decrease between 2000 and 2010. The number of induced abortions decreased from 10,362 in 2000 to 6,738 in 2010. There was a statistically significant upward trend in abortion ratios in DTs 1 and 7 as well as a significant downward trend in DT 4. The other DTs presented stable trends of abortion ratio. Conclusion: Postabortion hospitalization rate and estimates of induced abortion were high in the state of Piaui, with a downward trend of induced abortion ratio for the study period.

Descriptors: Induced abortion; Hospitalization; Time Series Studies.
Artigo Original
1) Universidade Estadual do Piauí UESPI - Teresina (PI) - Brasil

Recebido em: 01/02/2015

Revisado em: 26/02/2015 Aceito em: 07/05/2015 


\section{RESUMEN}

Objetivo: Estimar el aborto provocado y evaluar su tendencia en el estado de Piaui y en sus territorios de desarrollo (TD) entre el período de 2000 y 2010. Métodos: Se realizó un estudio ecológico, de serie temporal utilizando datos secundarios de ingresos hospitalarios por complicaciones del aborto entre febrero y junio de 2014. La estimación de los abortos inducidos fue calculada a través de la metodología del Instituto Guttmacher. Se aplicó el método Joinpoint para la identificación de los cambios significativos de las tendencias. Resultados: Se analizaron 55.678 ingresos hospitalarios por aborto y se verificó una disminución del 35\% entre 2000 y 2010. El número de abortos inducidos disminuyo en Piaui en los extremos de la serie temporal variando de 10.362 en 2000 para 6.738 en 2010. Hubo una tendencia de aumento significativo de las razones de aborto en los TD 1 y 7 asi como una tendencia de reducción significativa del TD 4. Los otros TD presentaron una tendencia estable de la razón del aborto. Conclusión: La tasa de ingreso pos-aborto y las estimaciones de aborto inducido fueron elevadas en el estado de Piaui con una tendencia de disminución de la razón del aborto inducido en el período estudiado.

Descriptores: Aborto Inducido; Hospitalización; Estudios de Series Temporales.

\section{INTRODUÇÃO}

O aborto inseguro é um importante problema de saúde pública no mundo. Estima-se que, em 2008, ocorreram 43,8 milhões de abortos induzidos, $49 \%$ dos quais foram inseguros $^{(1)}$. Como consequência, houve 47.000 mortes por aborto nesse mesmo ano ${ }^{(2)}$. Apesar da magnitude, a mensuração da incidência do aborto e suas complicações é difícil de ser feita, notadamente em países onde as leis são restritivas, como é o caso do Brasil. Uma das grandes dificuldades encontradas nas pesquisas sobre aborto é a tendência de omissão de sua prática pelas mulheres, geralmente decorrente do estigma e do receio da denúncia $^{(3)}$. Mesmo com a garantia de confidencialidade, muitas delas alegam aborto espontâneo quando perguntadas diretamente ${ }^{(4,5)}$.

No Brasil, as pesquisas sobre magnitude do aborto foram feitas, principalmente, a partir de métodos indiretos. Em 1991, o Instituto Alan Guttmacher estimou a ocorrência de 1.443 .350 abortos provocados no país, com taxa anual de 3,65 abortos para cada 100 mulheres de 15 a 49 anos. O cálculo baseou-se no número oficial de mulheres internadas por complicações de aborto e para curetagem uterina, aplicando-se fatores de correção para estimar o aborto $\operatorname{provocado}^{(6,7)}$.
Os dados baseados em internações para curetagem uterina do Sistema Único de Saúde (SUS) foram atualizados em 2006. Em todos os grupos etários de 15 a 49 anos, houve redução no número de internações por aborto entre 1992 (344.956 internações) e 2005 (250.447 internações). Dessa forma, as estimativas de aborto provocado também decresceram de 1.455.283 abortos em 1992 para 1.056.573 em 2005. As taxas de aborto inseguro para cada 100 mulheres das regiões Norte e Centro-Oeste foram mais que o dobro das observadas na região $\mathrm{Sul}^{\left({ }^{(8)} \text {. }\right.}$

A principal crítica às estimativas de aborto realizadas por técnicas indiretas, como os cálculos obtidos por meio do número de internações hospitalares, é o fato de que somente representam as mulheres que tiveram complicações e necessitaram de assistência hospitalar pública ${ }^{(9)}$. Se, por um lado, há possibilidade de subestimativa da magnitude, por outro, os métodos indiretos têm a vantagem de ser viáveis do ponto de vista financeiro e metodológico, principalmente por contornar a dificuldade do relato da prática do aborto pelas mulheres ${ }^{(10)}$.

Em 2010, um levantamento por amostragem randomizada e domiciliar utilizou a técnica de urna para traçar a magnitude do aborto no Brasil urbano, inclusive pequenos municípios, abrangendo 2.002 mulheres alfabetizadas entre 18 e 39 anos. O levantamento demonstrou que $15 \%$ delas já tinham feito pelo menos um aborto e que, ao completar 40 anos, uma em cada cinco mulheres já havia provocado o aborto. Além disso, quase metade $(48 \%)$ daquelas que abortaram necessitava de internação hospitalar para tratar complicações da prática ${ }^{(11)}$.

Existem muitas lacunas quanto à segurança das evidências em estimativas sobre incidência e morbimortalidade relacionadas ao aborto inseguro no Brasil. Não se sabe se o comportamento dessa incidência e da morbimortalidade do aborto é o mesmo para mulheres de regiões diferentes do país, em contextos socioeconômicos diversos. Dados de outros estudos mostraram que são as mulheres pobres e menos escolarizadas as de maior vulnerabilidade aos riscos do aborto realizado de maneira insegura ${ }^{(12,13)}$. Este artigo apresentou as primeiras informações consolidadas sobre a magnitude do aborto provocado no estado do Piauí, por meio de técnicas diretas ou indiretas. Assim, o objetivo deste estudo foi estimar o aborto provocado e avaliar sua tendência no estado do Piauí e em seus territórios de desenvolvimento (TD) no período de 2000 a 2010.

\section{MÉTODOS}

Realizou-se estudo ecológico de série temporal da estimativa de abortos provocados, tendo como 
unidade de análise o estado do Piaú e seus territórios de desenvolvimento (TD) no período entre 2000 e 2010, sendo desenvolvido no período de fevereiro a junho de 2014.

O estado do Piauí é composto por 11 TD, englobando 224 municípios $^{(14)}$. O número de municípios e o percentual de mulheres em idade reprodutiva (15-44 anos) de cada TD são, respectivamente: TD1 - Planície Litorânea (11; 8,5\%); TD2 - Cocais (22; 11,3\%); TD 3 - Carnaubais (16; 4,8\%); TD 4 - Entre Rios $(31 ; 40,4 \%)$; TD 5 - Vale do Sambito (16; 3,5\%); TD6 - Vale do Rio Guaribas (39; 10,5\%); TD 7 - Vale do Canindé (17; 4,0\%); TD 8 - Serra da Capivara (18; 4,3\%); TD 9 - Vale dos rios Piauí e Itaueira (19; 4,6\%); TD 10 - Tabuleiros do Alto Parnaíba (12; 2,4\%); TD 11 Chapada dos Mangabeiras $(25 ; 5,7 \%)^{(15)}$.

Coletou-se o número de internações hospitalares de mulheres submetidas à curetagem uterina por consequência de aborto, obtido no Sistema de Informação Hospitalar (SIH/SUS), do Departamento de Informática do SUS (DATASUS). O número de nascidos vivos, disponibilizado pelo Sistema de Informações sobre Nascidos Vivos (SINASC) e pela Secretaria de Saúde do Piauí, foi coletado de cada município e, posteriormente, tabulado para cada território de desenvolvimento. Além disso, os dados referentes ao número de mulheres por faixa etária e por cidade foram obtidos do Censo Demográfico de 2000 e de 2010, realizado pelo Instituto Brasileiro de Geografia e Estatística (IBGE).

Fez-se a procura do Código Internacional de Doenças (CID-10), capítulo XV (que trata de Gravidez, Parto e Puerpério), e também do código da tabela de procedimento hospitalar para curetagem uterina por aborto (0411020013) do SUS na base de dados do SIH/SUS. Utilizaram-se os CIDs O03 (aborto espontâneo), O04 (aborto por razões médicas e legais), O05 (outros tipos de aborto), O06 (aborto não especificado) e O07 (falha na tentativa de aborto) para a coleta de dados.

As estimativas de aborto induzido foram baseadas em metodologia empregada pelo Instituto Guttmacher, já utilizada em outros estudos brasileiros ${ }^{(6-8,16)}$. Calculou-se a estimativa através da fórmula: número total de abortos provocados $=$ número total de internações por curetagem uterina $x(0,75)^{\mathrm{i}} x(1,125)^{\mathrm{ii}} \times(2)^{\mathrm{iii}}$. A metodologia do Instituto Guttmacher considera a necessidade de três fatores de correção, presumindo que: i) $25 \%$ das internações hospitalares por complicações de aborto seriam decorrentes de aborto espontâneo, de modo que o número total deve ser multiplicado por 0,75 ; ii) $12,5 \%$ dos casos poderiam ser de sub-registros (mulheres internadas com outros diagnósticos ou em locais fora do Sistema Único de Saúde), devendo o número de internações ser multiplicado por 1,125; e iii) as mulheres hospitalizadas para curetagem representam $50 \%$ das que induziram o aborto, ou seja, para cada mulher internada, há pelo menos uma outra que não precisou ou não foi ao hospital, de forma que o número total de internações deve ser multiplicado por 2. Utilizou-se o fator de correção 2 porque, segundo a Pesquisa Nacional do Aborto, $48 \%$ das mulheres que disseram ter provocado o aborto precisaram de internação hospitalar para curetagem uterina ${ }^{(11)}$.

Com a estimativa de abortos induzidos estabelecida, foi obtida a razão de abortos induzidos segundo o número de nascidos vivos, multiplicando-se por 100 , de acordo com a fórmula: razão de aborto induzidos = número de abortos induzidos/número de nascidos vivos $x 100^{(10)}$.

Para os anos 2000 e 2010, calcularam-se as taxas de internação por complicações do aborto, padronizadas pelo número de mulheres em idade reprodutiva (de $15 \mathrm{a}$ 44 anos) de cada cidade, de acordo com a fórmula: taxa de internação por complicações de aborto $=$ número de mulheres internadas por complicações de aborto/número de mulheres em idade fértil x $1.000^{(10)}$.

Avaliou-se a tendência da razão de aborto induzido utilizando-se o método Joinpoint, baseado em software desenvolvido pelo Instituto Nacional do Câncer dos Estados Unidos ${ }^{(17)}$. O Joinpoint permite o ajuste de dados de uma série a partir do menor número possível de joinpoints (zero, ou seja, uma reta sem pontos de inflexão) e testa se a inclusão de joinpoints é estatisticamente significativa. Os testes de significância estatística utilizados basearam-se no método de permutação de Monte Carlo e no cálculo da variação percentual anual da razão, utilizando o logaritmo da mesma ${ }^{(18)}$. Quando houve mudança, cada ponto significativo na tendência foi mantido no modelo final. Para descrever a tendência linear por período, calcularamse a variação percentual anual (VPA) e seus respectivos intervalos de confiança de 95\% (IC 95\%), compondo uma linha de regressão de acordo com o logaritmo natural das taxas. Considerou-se que ocorreu aumento na razão de aborto quando a tendência foi de crescimento e o valor mínimo do intervalo de confiança foi superior a zero $(>0)$. Por outro lado, considerou-se que ocorreu redução quando houve declínio na tendência e o valor máximo do intervalo de confiança foi inferior a zero $(<0)$. A estabilidade foi definida quando, independentemente da tendência, o intervalo de confiança incluiu o valor zero.

Apesar de trabalhar com dados secundários e de domínio público, o estudo foi aprovado pelo Comitê de Ética em Pesquisa da Universidade Estadual do Piauí (parecer n. ${ }^{\circ}$ 44614).

\section{RESULTADOS}

Entre 2000 e 2010, 55.678 mulheres foram tratadas nos hospitais do Piauí por complicações de aborto. Em todo o estado, houve diminuição no número de internações para 
realização de curetagem uterina, de 6.140 em 2000 para 3.993 em 2010 - um declínio de 35\% entre os extremos da série histórica. Entre 2002 e 2005, ocorreu flutuação nesse número, mas a partir de 2006 se observa lenta e gradativa diminuição das internações (Figura 1). O TD 4, onde se encontra a capital do estado, Teresina, apresentou queda da taxa de internação padronizada por 1.000 mulheres de 15-44 anos, passando de 16,7 em 2000 para 6,7 em 2010. Nos TDs 1, 7, 8, 9, 10 e 11, houve aumento das taxas de internação (Figura 2).

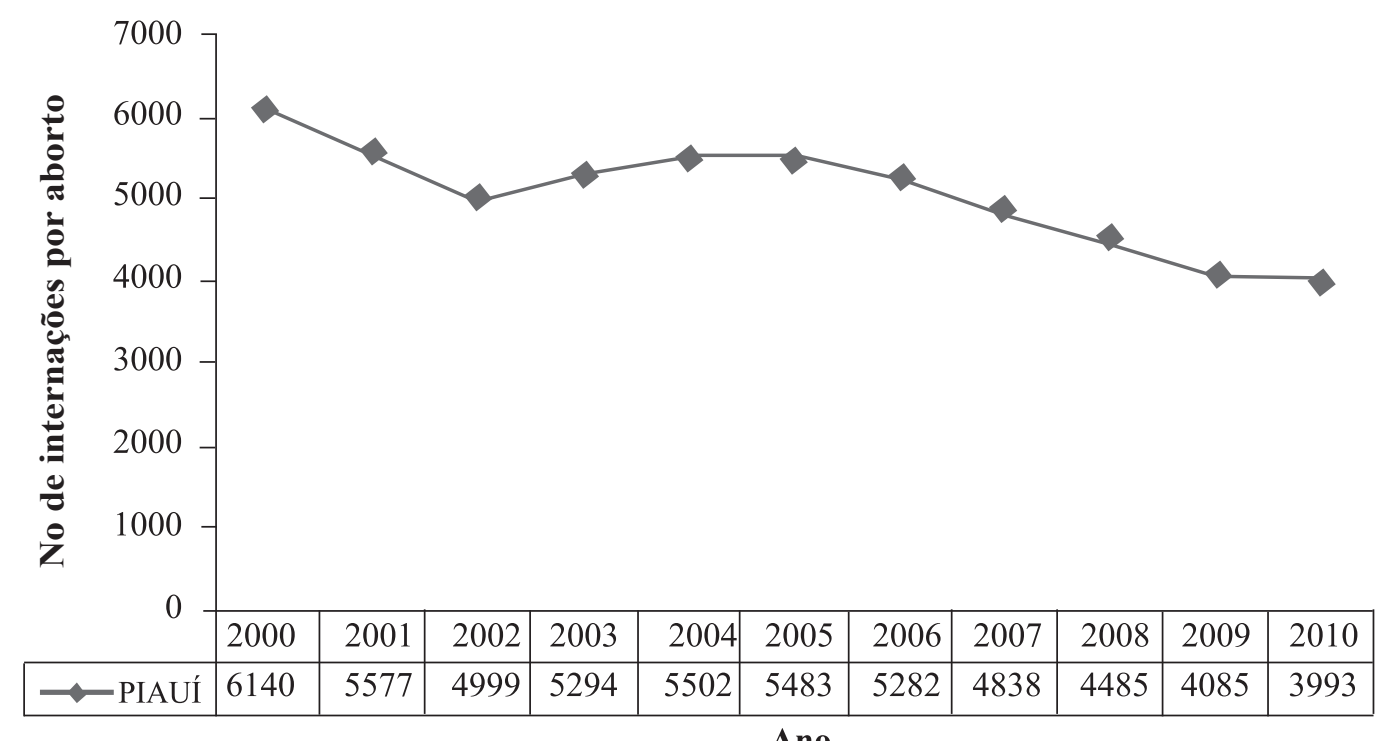

Figura 1 - Número de internações por complicações de ano aborto no estado do Piauí, nos anos 2000 a 2010. Piauí, 2014.

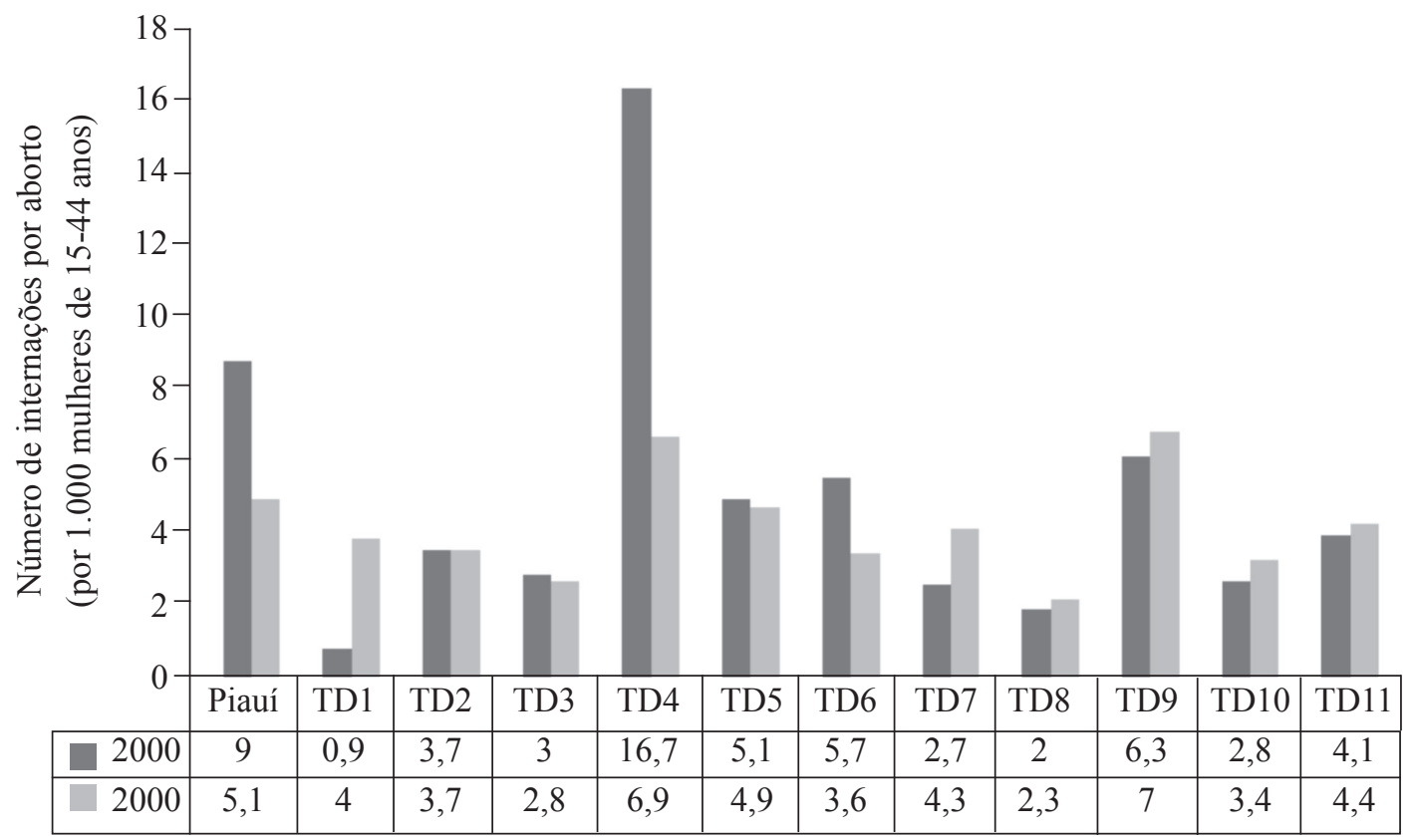

Figura 2 - Taxa de internações por complicações de aborto de acordo com os territórios de desenvolvimento no estado do Piauí, nos anos 2000 a 2010. Piauí, 2014. 
Tabela I - Estimativa de abortos induzidos de acordo com territórios de desenvolvimento (TD) no estado do Piauí, nos anos 2000 a 2010. Piauí, 2014.

\begin{tabular}{|c|c|c|c|c|c|c|c|c|c|c|c|c|}
\hline \multirow{2}{*}{ TD } & 2000 & 2001 & 2002 & 2003 & 2004 & 2005 & 2006 & 2007 & 2008 & 2009 & 2010 & TOTAL \\
\hline & $\mathbf{n}$ & $\mathbf{n}$ & $\mathbf{n}$ & $\mathbf{n}$ & n & $\mathbf{n}$ & $\mathbf{n}$ & $\mathbf{n}$ & $\mathbf{n}$ & $\mathbf{n}$ & $\mathbf{n}$ & $\mathrm{n} / \%$ \\
\hline Todos & 10.362 & 9.412 & 8.436 & 8.935 & 9.288 & 9.252 & 8.914 & 8.165 & 7.568 & 6.893 & 6.738 & $93.963 / 100$ \\
\hline TD1 & 87 & 182 & 230 & 203 & 125 & 467 & 466 & 628 & 474 & 505 & 440 & $3.807 / 4,0$ \\
\hline TD2 & 473 & 633 & 584 & 600 & 741 & 705 & 668 & 606 & 432 & 380 & 548 & $6.370 / 6,8$ \\
\hline TD3 & 186 & 194 & 98 & 122 & 275 & 187 & 255 & 213 & 174 & 172 & 177 & $2.053 / 2,2$ \\
\hline TD4 & 7.752 & 6.723 & 5.675 & 6.244 & 6.337 & 5.986 & 5.311 & 4.632 & 4.352 & 3.839 & 3.635 & $60.486 / 64,4$ \\
\hline TD5 & 224 & 162 & 140 & 127 & 157 & 152 & 138 & 133 & 172 & 138 & 224 & $1.767 / 1,9$ \\
\hline TD6 & 717 & 538 & 545 & 521 & 574 & 486 & 579 & 604 & 646 & 604 & 493 & $6.307 / 6,7$ \\
\hline TD7 & 128 & 160 & 169 & 160 & 149 & 172 & 221 & 184 & 223 & 184 & 226 & $1.976 / 2,1$ \\
\hline TD8 & 103 & 137 & 116 & 140 & 203 & 305 & 370 & 311 & 189 & 169 & 132 & $2.175 / 2,3$ \\
\hline TD9 & 363 & 334 & 454 & 420 & 289 & 304 & 461 & 491 & 489 & 467 & 424 & $4.496 / 4,8$ \\
\hline TD10 & 74 & 73 & 126 & 103 & 108 & 113 & 116 & 127 & 103 & 91 & 108 & $1.142 / 1,2$ \\
\hline TD11 & 255 & 276 & 299 & 295 & 330 & 375 & 329 & 236 & 314 & 344 & 331 & $3.384 / 3,6$ \\
\hline
\end{tabular}

TD: territórios de desenvolvimento.

Tabela II - Razão de abortos induzidos, de acordo com territórios de desenvolvimento no estado do Piaú, nos anos 2000 a 2010. Piauí, 2014.

\begin{tabular}{lccccccccccc}
\hline TD & $\mathbf{2 0 0 0}$ & $\mathbf{2 0 0 1}$ & $\mathbf{2 0 0 2}$ & $\mathbf{2 0 0 3}$ & $\mathbf{2 0 0 4}$ & $\mathbf{2 0 0 5}$ & $\mathbf{2 0 0 6}$ & $\mathbf{2 0 0 7}$ & $\mathbf{2 0 0 8}$ & $\mathbf{2 0 0 9}$ & $\mathbf{2 0 1 0}$ \\
\hline Todos & 17,6 & 16,1 & 14,9 & 16,2 & 16,9 & 16,3 & 16,1 & 13,8 & 14,3 & 13,5 & 13,5 \\
TD1 & 1,7 & 3,8 & 4,7 & 4,2 & 2,6 & 9,5 & 9,5 & 13,8 & 10,1 & 11,4 & 10,7 \\
TD2 & 6,1 & 8,2 & 7,8 & 8,4 & 10,6 & 9,6 & 9,4 & 9,2 & 6,6 & 6,3 & 9,2 \\
TD3 & 6,5 & 6,2 & 3,3 & 4,2 & 9,7 & 6,1 & 8,8 & 7,5 & 5,9 & 6,2 & 6,4 \\
TD4 & 33,6 & 30,9 & 27,8 & 31,2 & 31,8 & 29,3 & 26,2 & 24,0 & 21,9 & 20,1 & 19,2 \\
TD5 & 10,7 & 7,8 & 7,2 & 7,1 & 8,7 & 8,3 & 7,7 & 7,8 & 10,0 & 8,6 & 16,0 \\
TD6 & 11,8 & 8,5 & 9,0 & 9,0 & 10,1 & 8,1 & 10,1 & 10,6 & 12,0 & 11,5 & 9,4 \\
TD7 & 5,7 & 7,2 & 7,4 & 7,4 & 6,9 & 7,7 & 10,8 & 9,2 & 12,0 & 9,5 & 14,6 \\
TD8 & 5,7 & 6,1 & 4,6 & 5,6 & 8,5 & 11,6 & 15,8 & 13,0 & 8,0 & 7,2 & 6,0 \\
TD9 & 12,7 & 10,9 & 16,5 & 14,9 & 10,5 & 11,0 & 16,0 & 19,0 & 19,6 & 19,1 & 13,8 \\
TD10 & 5,4 & 4,6 & 8,8 & 6,9 & 6,7 & 7,2 & 7,5 & 8,1 & 7,3 & 6,3 & 7,7 \\
TD11 & 6,9 & 7,5 & 8,0 & 7,9 & 8,5 & 9,1 & 8,4 & 5,9 & 8,4 & 9,7 & 10,0 \\
\hline
\end{tabular}

TD: territórios de desenvolvimento.

Tabela III - Tendências da razão de aborto induzido, de acordo com territórios de desenvolvimento (TD) no estado do Piauí, nos anos 2000 a 2010. Piauí, 2014.

\begin{tabular}{lccccc}
\hline TD & RA - 2000 & RA - 2010 & VPA $\mathbf{( \% )}$ & IC 95\% & Tendência \\
\hline Todos & 17,6 & 13,5 & $-2,2^{*}$ & $(-3,5$ a $-0,9)$ & redução \\
TD1 & 1,7 & 10,7 & $+15,8^{*}$ & $(6,6$ a 25,9$)$ & aumento \\
TD2 & 6,1 & 9,2 & $+1,4$ & $(-5,3$ a 8,6$)$ & estável \\
TD3 & 6,5 & 6,4 & $+1,4$ & $(-4,9$ a 8,1$)$ & estável \\
TD4 & 33,6 & 19,2 & $-5,1^{*}$ & $(-7,1$ a $-2,9)$ & redução \\
TD5 & 10,7 & 16,0 & $+4,7$ & $(-3,5$ a 13,8$)$ & estável \\
TD6 & 11,8 & 9,4 & $+0,9$ & $(-1,9$ a 3,9) & estável \\
TD7 & 5,7 & 14,6 & $+7,9 *$ & $(4,7$ a 11,1$)$ & aumento \\
TD8 & 5,7 & 6,0 & $+3,4$ & $(-5,1$ a 12,8$)$ & estável \\
TD9 & 12,7 & 13,8 & $+3,6$ & $(-0,7$ a 8,2) & estável \\
TD10 & 5,4 & 7,7 & $+2,0$ & $(-1,7$ a 5,9) & estável \\
TD11 & 6,9 & 10,0 & $+2,4$ & $(-0,4$ a 5,3) & estável \\
\hline
\end{tabular}

TD: territórios de desenvolvimento; RA: razão de aborto; VPA: variação percentual anual; IC: intervalo de confiança

* Significativamente diferente de $0 \%(\mathrm{p}<0,05)$ 
A estimativa de abortos induzidos ocorridos no estado, separados por ano e território de desenvolvimento, é mostrada na Tabela I. Assim como nas internações, o número de abortos induzidos decresceu no Piauí entre os extremos da série temporal, passando de 10.362 em 2000 para 6.738 em 2010. Durante o período analisado, a estimativa de abortos induzidos aumentou em sete TDs $(1,2,7,8,9,10 \mathrm{e}$ 11), diminuiu em dois (4 e 6) e não apresentou mudança nos TDs 3 e 5 . O TD 4 concentrou $64,4 \%$ dos abortos induzidos estimados.

As Tabelas II e III mostraram a razão de abortos induzidos por 100 nascidos vivos e a tendência da razão de aborto induzido, respectivamente. Observou-se uma tendência de decréscimo significativa na razão de aborto em todo o estado (VPA = -2,2; IC 95\% -3,5 a -0,9; $p<0,05$ ). Em todo o período, observou-se uma tendência de aumento significativo nas razões nos TD 1 (VPA=15,8; IC 95\% 6,6 a $25,9 ; p<0,05)$ e 7 (VPA=7,9; IC 95\% 4,7 a 11,$1 ; p<0,05)$. $\mathrm{O}$ TD 4 demonstrou tendência de redução significativa (VPA $=-5,1$; IC $95 \%-7,1$ a $-2,9 ; p<0,05$ ). Os demais TDs $(2,3,5,6,8,9,10$ e 11) apresentaram tendência estável da razão de aborto.

\section{DISCUSSÃO}

Este estudo é o primeiro a estimar a incidência de aborto induzido no Piauí por meio do método de internações hospitalares. A obtenção de dados confiáveis sobre aborto induzido é limitada em todo o mundo, principalmente em locais onde a lei é restritiva, como o Brasil. Não existe método ideal para estimar o aborto induzido e, dessa forma, métodos diversos podem ser apropriados em diferentes contextos e objetivos. Mesmo em locais onde é permitido, o aborto tende a ser pouco declarado pelas mulheres quando se utilizam técnicas de pesquisa diretas, como entrevistas face a face ${ }^{(9,16,19)}$.

A pesquisa no Piauí evidenciou diminuição no número de internações por complicações de aborto nos anos 20002010, com decréscimo significativo na tendência da razão de aborto induzido. A redução na incidência e razão de aborto também foi observada no TD 4, onde se concentra a maior parcela das mulheres em idade reprodutiva do estado. A maioria dos TD apresentou leve aumento nas razões de aborto, mas sem significância estatística. Padrão semelhante foi verificado em estudo conduzido no estado de Pernambuco, que utilizou o método das internações hospitalares (porém com multiplicador 5) de 1996 a 2006. Em Pernambuco, a estimativa de abortos inseguros se apresentou elevada no período estudado, mas com tendência de diminuição da razão de aborto no estado como um todo ${ }^{(16)}$.
No Brasil, o número de internações para tratamento de complicações do aborto vem diminuindo desde a década de 1990. Um estudo mostrou que houve redução de $41 \%$ nas internações, passando de 282.000 em 1992 para $165.000 \mathrm{em}$ 2009. Apesar da diminuição, as regiões Norte e Nordeste ainda concentraram o maior número de internações. A taxa de internações por 1.000 mulheres de 15-44 anos foi de 2,8 em todo o Brasil e de 3,7 no Nordeste ${ }^{(19)}$. Mesmo com a redução das taxas de internação, quase todos os territórios do Piauí apresentaram, em 2010, índices superiores aos observados no Brasil e na região Nordeste.

Os dados da pesquisa mostraram que a estimativa do número de abortos no Piauí diminuiu no período estudado, assim como da tendência da razão de aborto para todo o estado. Somente dois TD apresentaram tendência de aumento da razão de aborto induzido. Pelo menos duas explicações para esses achados precisam ser consideradas. Em primeiro lugar, é possível que tenha ocorrido diminuição do número de gestações indesejadas por causa do maior e mais correto uso de métodos contraceptivos eficazes. Dados da Pesquisa Nacional de Demografia e Saúde demonstram queda da proporção de gestações indesejadas no Brasil, passando de 23,1\% em 1996 para 18,2\% em 2006. Entre as regiões, o Nordeste apresentou, em 2006, a maior redução na proporção de gestações não desejadas, mas foram as mulheres pobres, menos escolarizadas e mais velhas que tiveram maior número de nascimentos não desejados. Além disso, houve aumento do uso de anticoncepcional oral em todas as classes econômicas, além da esterilização masculina e utilização do preservativo ${ }^{(20)}$.

Em segundo lugar, é preciso considerar que as mulheres passaram a utilizar métodos abortivos mais seguros, necessitando menos da internação hospitalar para tratar complicações. A partir da década de 1990, o misoprostol, também conhecido como Cytotec ${ }^{\circledR}$, tornouse o método abortivo predominante entre as mulheres brasileiras ${ }^{(21-23)}$. O crescimento de vendas do misoprostol nas farmácias, associado com o maior uso pelas mulheres, foi simultâneo à menor incidência de casos mais graves de complicações, principalmente infecciosas. Em Recife, um estudo retrospectivo avaliou 1.840 mulheres internadas para realização de curetagem uterina por aborto, observando taxa de infecção de 4,2\% nas usuárias de misoprostol e $49,4 \%$ naquelas que utilizaram outros métodos ${ }^{(24)}$. Pesquisa conduzida na República Dominicana também observou associação entre aumento de vendas do misoprostol e redução de complicações e mortalidade feminina relacionadas ao aborto inseguro. Neste país, onde o aborto é ilegal, as internações diminuíram de 11,7\% em 1986 para $1,7 \%$ em 2001, quando a droga foi introduzida no país ${ }^{(25)}$.

Se a hipótese do uso do misoprostol for verdadeira para a maioria das mulheres internadas, pode-se considerar 
que houve falha na sua eficácia. Quando utilizado de maneira correta, é um meio eficiente, seguro e de baixo custo para realização do aborto, determinando maior índice de abortos completos e, assim, menor necessidade de internação hospitalar para curetagem uterina ${ }^{(26)}$. Tendo em vista que a comercialização da droga é proibida no país e, por isso, obtida de forma clandestina pelas mulheres, há possibilidade do uso de subdoses ou que o próprio princípio ativo seja falsificado(27). A informação sobre como utilizar a medicação também é frequentemente obtida de outras mulheres, vendedores de farmácias e sites da internet, o que pode contribuir para a ineficácia ${ }^{(27,28)}$. Além disso, muitas mulheres procuram os serviços de saúde pelos efeitos colaterais da medicação, como cólicas e sangramento, e ainda pela dúvida em saber se o aborto foi completo ${ }^{(29)}$.

Este estudo apresentou pelo menos duas limitações. Primeiro, o método das internações hospitalares, baseado em dados de mulheres hospitalizadas por complicações do aborto, teve como desvantagem a seleção amostral, que não considera mulheres que não necessitaram de internação após o aborto. Mesmo sendo considerada ferramenta útil para mensurar a incidência de aborto induzido em uma grande população, uma das maiores críticas é que o método pode subestimar ou superestimar estes achados a depender do fator multiplicador utilizado, que deve ser baseado na realidade de cada localidade ${ }^{(5,10)}$. Segundo, a utilização do fator multiplicador 2 foi baseada na única pesquisa de abrangência nacional pelo método direto já realizada ${ }^{(11)}$. No entanto, este estudo somente entrevistou mulheres urbanas. Não avaliou a incidência de abortos e tampouco mostrou o panorama do aborto nas diferentes regiões do país.

\section{CONCLUSÃO}

A taxa de internação pós-aborto e as estimativas de aborto induzido foram elevadas no estado do Piauí, com tendência de diminuição da razão de aborto induzido no período estudado.

Outras pesquisas são necessárias para estimar o aborto induzido com métodos diretos, além de conhecer as formas e percursos para abortar das mulheres no Piauí.

\section{REFERÊNCIAS}

1 Sedgh G, Singh S, Shah IH, Ahman E, Henshaw SK, Bankole A. Induced abortion: incidence and trends worldwide from 1995 to 2008. Lancet. 2012;379(9816):625-32.

2. World Health Organization - WHO. Unsafe abortion: global and regional estimates of the incidence of unsafe abortion and associated mortality in 2008. 6th ed. Geneva: World Health Organization; 2011.
3. Jones RK, Hill KH. Underreporting of induced and spontaneous abortion in the United States: an analysis of the 2002 National Survey of Family Growth. Stud Fam Plann. 2007;38(3):187-97.

4. Shellenberg KM, Moore AM, Bankole A, Juarez F, Omideyi AK, Palomino N, et al. Social stigma and induced abortion: results from an exploratory study. Glob Public Health. 2011;6(Suppl 1):S111-25.

5. Okonofua F, Omo-Aghoja L, Bello Z, Osughe M, Agholor K. Self-reporting of induced abortion by women attending prenatal clinics in urban Nigeria. Int J Obstet Gynecol. 2010;111(2):122-5.

6. Singh S, Wulf D. Estimating abortion levels in Brazil, Colombia and Peru, using hospital admissions and fertility data. Int Fam Plan Perspect. 1991;17(1):8-24.

7. Singh S, Wulf D. Estimated levels of induced abortion in six Latin American countries. Int Fam Plan Perspect. 1994;20(1):4-13.

8. Monteiro M, Adesse L. Estimativas de aborto induzido no Brasil e Grandes Regiões, 1992-2005. Anais do XV Encontro Nacional de Estudos Populacionais, Caxambú-MG; 2006 Set 18-22. Caxambu: Ações Afirmativas em Direitos e Saúde; 2006. p. 1-10.

9. Rossier C. Estimating induced abortion rates: a review. Stud Fam Plann. 2003;34(2):87-102.

10. Singh S, Remez L, Tartaglione A. Methodologies for estimating abortion incidence and abortion-related morbidity: a review. New York: Guttmacher Institute; Paris: International Union for Scientific Study of Population; 2010.

11. Diniz D, Medeiros M. Pesquisa Nacional de Aborto: um estudo com técnica de urna. Ciênc Saúde Coletiva. 2010;15(Supl 1):S959-66.

12. Souza MG, Fusco CL, Andreoni SA, Souza, Silva R. Prevalência e características sociodemográficas de mulheres com aborto provocado em uma amostra da população da cidade de São Paulo, Brasil. Rev Bras Epidemiol. 2014;17(2):297-312.

13. Cecatti JG, Guerra GVQL, Sousa MH, Menezes GMS. Aborto no Brasil: um enfoque demográfico. Rev Bras Ginecol Obstet. 2010;32(3):105-11.

14. Governo do Estado do Piauí. Lei Complementar $\mathrm{n}^{\mathrm{o}}$ 87, de 22 de agosto de 2007 [legislação na internet]. Estabelece o Planejamento Participativo Territorial para o Desenvolvimento Sustentável do Estado do Piauí e dá outras providências. Teresina: Governo de Estado do Piauí [acesso em 2014 Dez 12]. Disponível 
em: http://www.seplan.pi.gov.br/uapr/lei87_22-082007.pdf

15. Instituto Brasileiro de Geografia e Estatística - IBGE. Censo Demográfico 2000 e 2010. Características da População e dos Municípios [Internet]. Rio de Janeiro: IBGE; 2010 [acesso em 2014 Dez 12]. Disponível em: http:/www.sidra.ibge.gov.br/cd/cd2010universo. asp? $=7 \& \mathrm{i}=\mathrm{P}$

16. Mello FMB, Sousa JL, Figueroa JN. Magnitude do aborto inseguro em Pernambuco, Brasil, 1996 a 2006. Cad Saúde Pública. 2011;27(1):87-93.

17. National Cancer Institute. Joinpoint Regression Program. Version 3.4.3 [Internet]. Bethesda; 2013 [acesso em 2014 Jan 30]. Disponível em: http:// surveillance.cancer.gov/joinpoint/

18. Kim HJ, Fay MP, Feuer EJ, Midthune DN. Permutation tests for joinpoint regression with applications to cancer rates. Stat Med. 2000;19(3):335-51.

19. Ministério da Saúde (BR), Centro Brasileiro de Análise e Planejamento. Pesquisa Nacional de Demografia e Saúde da Criança e da Mulher - PNDS 2006: dimensões do processo reprodutivo e da saúde da criança. Brasília: Ministério da Saúde; 2009.

20. Singh S, Monteiro MFG, Levin J. Trends in hospitalization for abortion-related complications in Brazil, 1992-2009: why the decline in numbers and severity? Int J Gynecol Obstet. 2012;118(Suppl 2):S99-S106.

21. Barbosa R, Arilha M. The Brazilian experience with Cytotec. Stud Fam Plann. 1993;24(4):236-40.

22. Fonseca W, Misago C, Correia LL, Parente JAM, Oliveira FC. Determinantes de aborto provocado entre mulheres admitidas em hospitais em localidade da região Nordeste do Brasil. Rev Saúde Pública. 1996;30(4):13-8.
23. Fonseca W, Misago C, Freitas P, Santos E, Fernandes L, Correa L. Características sócio-demográficas, reprodutivas e médicas de mulheres admitidas por aborto em hospital da Região Sul do Brasil. Cad Saúde Pública. 1998;14(2):279-86.

24. Faúndes A, Santos LC, Carvalho M, Gras C. Postabortion complications after interruption of pregnancy with misoprostol. Adv Contracept. 1996;12(1):1-9.

25. Miller S, Lehman T, Campbell T, Hemmerling A, Anderson SB, Rodriguez H, et al. Misoprostol and declining abortion-related morbidity in Santo Domingo, Dominican Republic: a temporal association. BJOG. 2005;112(9):1291-6.

26. Kapp N, White P, Tang J, Jackson E, Brahmi D. A review of evidence for safe abortion. Contraception. 2013;88(3):350-63.

27. Diniz D, Madeiro A. Cytotec e aborto: a polícia, os vendedores e as mulheres. Ciênc Saúde Coletiva. 2012;17(7):1795-803.

28. Diniz D, Medeiros M. Itinerários e métodos do aborto ilegal em cinco capitais brasileiras. Ciênc Saúde Coletiva. 2012;17(7):1671-81.

29. Zamberlin N, Romero M, Ramos S. Latin American women's experiences with medical abortion in settings where abortion is legally restricted. Reprod Health. 2012;9(1):34.

\section{Endereço para correspondência:}

Alberto Madeiro

Rua Olavo Bilac, 2335

Bairro: Centro/Sul

CEP: 64049-550 - Teresina - Piauí - Brasil

E-mail:madeiro@uol.com.br 